\title{
La Relación entre las Estrategias de Innovación: Coexistencia o Complementariedad ${ }^{*}$
}

\author{
Jaider Vega-Jurado, Antonio Gutiérrez-Gracia, Ignacio Fernández-de-Lucio '
}

\begin{abstract}
This paper explores the relationships between internal R\&D and external knowledge acquisition as business innovation strategies. Drawing on absorptive capacity framework (Cohen and Levinthal, 1990), we distinguish and empirically evaluate two possible relationships: coexistence and complementarity. The analysis is based on a large sample of manufacturing firms taken from the Spanish Survey of Technological Innovation 2004. Our results show coexistence between internal R\&D and external knowledge acquisition but they do not provide evidence that these activities are complementary with respect to influencing innovation performance. In other words, although our results suggest that internal R\&D activities are associated with a greater use of external knowledge sourcing strategies, they do not seem to have synergistic effects to develop new products.
\end{abstract}

Keywords: External knowledge acquisition; internal R\&D; complementarity; coexistence.

\section{Resumen}

Este artículo analiza las relaciones existentes entre la generación y la adquisición externa de conocimiento como estrategias de innovación empresarial. Para ello, y utilizando el concepto de capacidad de absorción (Cohen y Levinthal, 1990) como marco de referencia, se distinguen y se analizan empíricamente dos posibles relaciones: coexistencia y complementariedad. El estudio es realizado sobre una muestra amplia de empresas manufactureras españolas, empleando los datos derivados de la Encuesta sobre Innovación Tecnológica del año 2004. Los resultados muestran que en el contexto analizado, las actividades internas de I+D y las estrategias asociadas con la adquisición externa de conocimiento son estrategias coexistentes, pero no complementarias. En otras palabras, si bien las actividades internas de I+D facilitan la identificación y adquisición de conocimiento externo, no se encuentra evidencia a favor de que dichas actividades tengan un efecto sinérgico para el desarrollo de nuevos productos.

Palabras claves: Adquisición externa de conocimiento; I+D interna; complementariedad; coexistencia.

\footnotetext{
* Este artículo es resultado del proyecto ECO2008-0523 I/ECON financiado por el Ministerio de Ciencia e Innovación de España.

I INGENIO (CSIC - UPV), Instituto de Gestión de la Innovación y del Conocimiento. Ciudad Politécnica de la Innovación, Camino de Vera s/n, Edificio 8E; 46022, Valencia, España. Tel: +34 963877 048; fax: +34 96387709 I. E-mails: javega@ingenio.upv.es (J. VegaJurado); agutierr@ingenio.upv.es (A. Gutiérrez-Gracia); ifernand@ingenio.upv.es (I. Fernandez-de-Lucio).
} 


\section{Introducción}

La búsqueda y adquisición de conocimiento tecnológico ha emergido como un aspecto clave y complejo dentro de la estrategia de innovación empresarial. Las empresas no sólo tienen que decidir entre generar internamente el conocimiento que requieren $\circ$ adquirirlo de fuentes externas, sino además seleccionar el mecanismo más adecuado que le permita acceder a dicho conocimiento. Estos mecanismos han sido referidos en la literatura como estrategias de innovación o estrategias tecnológicas, y su análisis se ha convertido en un tema de gran interés entre los académicos de la innovación. En esta línea, una de las cuestiones que ha despertado interés en las últimas décadas ha sido el análisis de las relaciones existentes entre las estrategias de innovación. De hecho, podría decirse que desde que Cohen y Levinthal (1990) propusieron el concepto de capacidad de absorción, han aumentado los trabajos empíricos que exploran las relaciones existentes entre el desarrollo interno de actividades de I+D y la adquisición externa de conocimiento. Atendiendo a este concepto se ha argumentado que los esfuerzos que realiza la empresa para la creación de nuevo conocimiento no sólo estimulan el uso de fuentes externas de conocimiento, sino que además incrementan su capacidad para explotarlas eficientemente en el desarrollo de nuevos productos o procesos. En otras palabras, se asume que el desarrollo interno y la adquisición externa de conocimiento pueden llegar a ser estrategias complementarias para promover el desempeño innovador de la empresa.

No obstante, a pesar de lo extendido que está el argumento anterior, lo cierto es que la evidencia empírica no es concluyente al respecto, entre otras cosas, porque los autores han abordado el análisis de estas relaciones de forma muy diferente. En algunos casos la relación de complementariedad ha sido justificada a partir de los resultados de modelos de regresión que incluyen una estrategia como variable dependiente y otra como variable explicativa, mientras que en otros casos, la justificación se basa en los resultados de los análisis de correlación entre las variables. Este tipo de análisis, aunque demuestran que la adquisición externa y la generación interna de conocimiento pueden coexistir dentro de la estrategia global de la empresa, no constituyen evidencia directa a favor de la existencia de complementariedad entre las mismas (Cassiman y Veugelers,
2006). La complementariedad implica mucho más que la adopción conjunta de diferentes estrategias; supone la existencia de un efecto sinérgico entre ellas, en la medida en que la adopción de una incrementa el retorno marginal de la otra (Milgrom y Roberts, 1990).

El objetivo de este artículo es, precisamente, analizar las relaciones existentes entre la generación y la adquisición externa de conocimiento como estrategias de innovación empresarial, distinguiendo claramente entre la "coexistencia" y la "complementariedad" como dos dimensiones que, en conjunto, explican dichas relaciones. Como estrategias de innovación se considera no sólo el desarrollo interno de actividades de I+D (estrategia de hacer), sino también un conjunto de actividades asociadas con la adquisición externa de conocimiento a través de transacciones de mercado (comprar) y a través de la cooperación con otros agentes (Cooperar). Con relación a esta última estrategia, se distingue, además, entre la cooperación que se establece con agentes industriales (proveedores, clientes, competidores) y la que se lleva a cabo con agentes institucionales (universidades, OPIs, centros tecnológicos). Esta última distinción es relevante, dado que el conocimiento derivado de cada uno de estos tipos de agentes tiende a ser de diferente naturaleza, por lo que no sólo pueden afectar de forma distinta el desempeño innovador de la empresa, sino que también pueden tener una relación diferente con las capacidades internas de la empresa. Por ejemplo, Cohen y Levinthal (1990) sugirieron que cuando la información relevante en el entorno está más asociada con la "ciencia básica"2, la empresa requerirá una mayor capacidad de absorción para explotarla debido a que no responde a sus necesidades prácticas.

El análisis es realizado sobre una muestra amplia de empresas manufactureras españolas que llevan a cabo actividades de innovación (33II empresas). Específicamente, los datos utilizados provienen de la Encuesta sobre Innovación Tecnológica en las Empresas para el año 2004 (EIT), llevada a cabo por el Instituto Nacional de Estadística de España (INE). El tamaño de la muestra y la diversidad de sectores industriales presentes en la misma permiten controlar el efecto que pueden ejercer dinámicas industriales específicas sobre las

\footnotetext{
${ }^{2}$ Estos autores emplean esta expresión para referirse fundamentalmente a las fuentes de conocimiento científico, tales como las universidades u organismos de investigación.
} 
relaciones entre las estrategias de innovación, lo cual constituye otro aspecto relevante de esta investigación.

El resto del artículo se estructura de la siguiente manera. En la siguiente sección se realiza una breve revisión de la literatura asociada con el análisis de las relaciones entre las estrategias de innovación. Posteriormente, se describen los aspectos metodológicos del estudio empírico, exponiendo los datos utilizados, y las técnicas de análisis empleadas, para luego proseguir con la presentación y discusión de los resultados. Por último, se presentan las principales conclusiones y se exponen algunas implicaciones políticas.

\section{Las relaciones entre las estrategias de innovación}

El estudio de las relaciones existentes entre las estrategias de innovación ha sido un tema que ha despertado el interés de los investigadores desde hace ya algún tiempo. Hay que señalar que los primeros trabajos realizados sobre este tema seguían la tradición derivada de la Teoría de los Costes de Transacción (TCT) ${ }^{3}$, la cual sugiere una completa sustitución entre las diferentes estrategias de innovación, por lo que por motivos de costes y riesgos la empresa debía elegir entre adoptar una u otra (Foray y Mowery, 1990). De esta forma, se consideraba que lo que afrontaba la empresa era básicamente un problema de selección entre alternativas sustitutivas, es decir, decidir entre hacer, comprar o cooperar como mecanismo para adquirir tecnología. Dentro de este marco conceptual, no se contemplaba la existencia de una relación entre las estrategias de innovación, más allá de su perfecta sustitución, y los análisis se centraban en determinar las condiciones bajo las cuales una estrategia era preferible sobre otra.

Mowery (1983), fue quizás el primer autor en sugerir la existencia de posibles relaciones entre las estrategias hacer $y$ comprar. En su estudio sobre los factores que influyen en la externalización de las actividades I+D, este autor no sólo señaló

\footnotetext{
${ }^{3}$ La TCT constituye uno de los enfoques más importantes para analizar lo que se ha denominado como las "fronteras de la empresa", es decir, la decisión empresarial entre el desarrollo interno o externo de una actividad. Esta teoría, ejemplificada principalmente por las contribuciones de Coase (1937) y Williamson (1975, 1985), tiene como objetivo principal identificar las fuentes de los costes de las transacciones que tienen lugar cuando "un bien o servicio es transferido a lo largo de una interfase tecnológica separable" (Williamson 198I) y, a partir de dicha identificación, especificar el mecanismo de gobierno que pueda hacerlas más eficientes.
}

elementos consistentes con el enfoque de la TCT (p.e. el efecto de la complejidad e interdependencia de las actividades de I+D), sino que además indicó que cuanto mayor es la capacidad tecnológica de una empresa (derivada del desarrollo interno de actividades de I+D) más probable es que ésta emplee la adquisición externa de tecnología como estrategia de innovación. Posteriormente, Mowery y Rosenberg (1989) volvieron a plantear una relación similar, pero esta vez entre la cooperación y el desarrollo interno de actividades de I+D. Estas argumentaciones van más allá de los fundamentos de la TCT e incluso la contradice en algunos aspectos. Si se tiene en cuenta que el desarrollo de actividades internas de I+D es una de las fuentes para el desarrollo de las competencias tecnológicas de la empresa, lo anterior supondría que las estrategias de hacer y comprar o hacer y cooperar más que opciones alternativas, pueden llegar a ser elementos complementarios en la estrategia de innovación empresarial. Esta relación está más cercana a los enfoques basados en las capacidades de la empresa ${ }^{4}$, los cuales desatacan la complementariedad tecnológica como la motivación principal para la adquisición externa de conocimiento.

El trabajo de Mowery abrió las puertas para que se estudiara no sólo los beneficios de cada estrategia particular, sino las potencialidades asociadas a su combinación dentro de la estrategia de innovación global de la empresa. Esta corriente tuvo un fuerte impulso gracias a la publicación del trabajo seminal de Cohen y Levinthal (1990) sobre la capacidad de absorción. Estos autores señalaron que las actividades internas de I+D cumplen un doble papel. Por una parte, constituyen una fuente de conocimiento importante para la obtención de ideas innovadoras $y$, por otra, incrementan la capacidad de la empresa para identificar, asimilar y explotar el conocimiento disponible fuera de sus fronteras, es decir, incrementan su capacidad de absorción. Partiendo de este concepto, se han desarrollado diversos estudios empíricos que analizan las relaciones existentes entre el know-how interno y externo o, en términos estratégicos, las relaciones entre las decisiones de hacer y comprar. Arora y Gambardella (1990, 1994), por ejemplo, encontraron que las grandes empresas con una mayor base de conocimiento son más activas en la búsqueda y adquisición de tecnología externa. En esta línea, Lowe y Taylor (1998) hallaron una relación similar entre la $I+D$ interna y la adquisición de

\footnotetext{
4 Dentro de este marco se destacan la teoría basada en los recursos -TBR- (Barney, 1991) y el enfoque de las capacidades dinámicas (Teece et al., 1997).
}

ISSN: 07I 8-2724. (http://www.jotmi.org)

Journal of Technology Management \& Innovation (C) Universidad Alberto Hurtado, Facultad de Economía y Negocios 
tecnología mediante licencias, mientras que Freeman (199l) indicó que las empresas que poseen un departamento de I+D tienden a usar más intensamente las fuentes externas de conocimiento.

Pero la relación entre las estrategias de innovación no sólo se da en el sentido anteriormente señalado. Para explotar eficientemente la tecnología adquirida, la empresa debe asimilarla e integrarla en sus procesos productivos, lo cual requiere estar en posesión de una buena base de conocimiento y habilidades tecnológicas. En este sentido, la adquisición de know-how externo, en lugar de sustituir, puede incentivar el desarrollo de actividades internas de I+D. Veugelers (1997), ofrece evidencia empírica a favor de esta última relación. Esta autora encontró que tanto la compra de tecnología como la cooperación, fomenta la I+D interna de la empresa, especialmente cuando ésta dispone de su propia infraestructura tecnológica (departamento de I+D con personal dedicado a tiempo completo). Kaiser (2002) y Becker y Dietz (2004) llegan también a resultados similares, y encuentran que las empresas que cooperan invierten más en actividades de $I+D$ que aquellas que no lo hacen.

En términos generales, existe evidencia empírica consistente a favor del papel que ejerce el conocimiento interno en la identificación y adquisición del conocimiento externo e, inversamente, del estímulo que otorga la adquisición externa de tecnología al desarrollo de actividades internas de I+D. Estos hallazgos corroboran la posibilidad que tiene la empresa para adoptar conjuntamente diversos mecanismos orientados a la obtención de tecnología, en otras palabras, que las estrategias hacer, comprar y cooperar coexisten en la estrategia global empresarial.

Los resultados anteriores han llevado a la emergencia de una nueva línea de investigación centrada en el análisis de complementariedades entre las diferentes estrategias de innovación. No obstante, hay que señalar que la investigación sobre este tema es aún incipiente y se enfrenta a dificultades metodológicas considerables (Athey and Stern, 1998).

Si bien la idea de complementariedad como tal no es reciente en el campo de la gestión estratégica, el análisis de complementariedades entre prácticas organizacionales no había sido objeto de exploración en la teoría económica de la empresa hasta que Milgrom y Roberts (1990) introdujeron y desarrollaron formalmente el concepto. Estos autores desarrollaron, además, una estilizada teoría matemática a partir de la cual establecen las condiciones necesarias para que dos actividades puedan ser consideradas complementarias. Esta teoría, conocida como la teoría de la supermodularidad, establece que dos actividades son complementarias si el cambio en la función de producción que se obtiene cuando las dos actividades se llevan a cabo de forma separada, es menor que el cambio resultante al desarrollar las dos actividades de forma conjunta.

Un aspecto importante que se desprende de los planteamientos de Milgrom y Roberts (1990), es que la complementariedad debe ser evaluada teniendo en cuenta una función de producción o de desempeño, lo cual, sin embargo, ha sido obviado en varios de los trabajos empíricos que abordan el estudio de la complementariedad entre las estrategias de innovación. En este campo una práctica muy común ha sido evaluar la complementariedad a través del enfoque de "correlación", propuesto por Arora y Gambardella $(1990)^{5}$. De esta forma, más que evidencia a favor de la complementariedad lo que realmente ofrecen es evidencia a favor de la coexistencia entre las estrategias.

Los trabajos de Laursen y Salter (2006) y Cassiman y Veugelers (2006), son algunos de los pocos que ofrecen evidencia empírica sobre la complementariedad entre la generación interna y la adquisición externa de conocimiento, entendida en el sentido descrito anteriormente.

Laursen y Salter (2006), analizan la relación entre el desempeño innovador de la empresa y el número de fuentes externas de conocimiento que ésta utiliza, considerando adicionalmente el efecto moderador de las actividades

\footnotetext{
${ }^{5}$ La amplia difusión que ha tenido el enfoque de correlación (también conocido como de adopción) como método para el análisis de la complementariedad obedece a su simplicidad y al hecho de que no requiere la utilización de una función de desempeño. Este último aspecto permite que el enfoque de correlación pueda ser utilizado, incluso, cuando sólo se dispone de información relacionada con la adopción de las diferentes estrategias o prácticas organizacionales. No obstante, se ha reconocido que este método sólo ofrece algunos indicios de complementariedad pero que difícilmente puede ser considerado como una prueba definitiva de su existencia. De hecho, si no se aseguran ciertas condiciones, el enfoque de correlación pude dar como resultado coeficientes de correlación positivos cuando no existe complementariedad, o por el contrario, encontrar una correlación negativa cuando en realidad las estrategias son complementarias (Athey y Stern, 1998)
}

ISSN: 07I 8-2724. (http://www.jotmi.org)

Journal of Technology Management \& Innovation (C) Universidad Alberto Hurtado, Facultad de Economía y Negocios 
internas de $1+D^{6}$. Estos autores llevan a cabo su análisis sobre una muestra de empresas manufactureras del Reino Unido y encuentran una relación curvilínea (en forma de $U$ invertida) entre el uso de fuentes externas de conocimiento y el desempeño innovador de la empresa, indicando con ello que la apertura hacía las ideas externas es sólo beneficiosa hasta cierto nivel. Adicionalmente, estos autores encuentran que la intensidad en I+D modera de forma negativa la relación entre el uso de las fuentes externas de conocimiento y el desempeño innovador de la empresa, lo cual asocian con un efecto de sustitución entre la generación interna y la adquisición externa de conocimiento.

Cassiman y Veugelers (2006), por su parte, analizan directamente la complementariedad entre las estrategias de innovación, focalizando su atención en las decisiones de hacer y comprar. Su estudio se realiza sobre una muestra de empresas manufactureras belgas y, a diferencia de Laursen y Salter (2006), encuentran que la generación y la adquisición externa de conocimiento tienen efectos complementarios sobre el desempeño innovador de la empresa. Este trabajo es uno de los más rigurosos desarrollados hasta la fecha, debido a que no sólo analiza la complementariedad entre las estrategias de innovación, sino que además examina las variables contextuales que afectan dicha complementariedad. De esta forma, los autores encuentran que la complementariedad entre las actividades internas de $I+D$ y la adquisición externa de conocimiento es sensible a la importancia que tienen las universidades y los institutos de investigación como fuente de información para los procesos de innovación. Su conclusión es que la complementariedad entre las estrategias hacer y comprar es mucho más fuerte en empresas que tienen una mayor dependencia hacía el know-how de carácter "básico", es decir, el derivado de agentes científicos como universidades e institutos de investigación.

Si bien los trabajos anteriores emplean métodos y variables diferentes, los dos tienen en común que analizan las relaciones entre la generación y la adquisición externa de conocimiento, teniendo en cuenta sus efectos sobre el desempeño innovador de la empresa. Aunque los resultados de estos trabajos no son directamente

\footnotetext{
${ }^{6}$ Nótese que estos autores no analizan específicamente las estrategias de adquisición de conocimiento externo, sino el uso de fuentes externas en general. No obstante, incluyen en su análisis el desarrollo de actividades internas de I+D. En este sentido, el análisis de estos autores constituye una mezcla entre estrategias de innovación y fuentes de conocimiento.
}

comparables, ponen de manifiesto que la complementariedad entre las estrategias de innovación no es un aspecto que deba darse por supuesto y que la misma puede depender de diferentes variables contextuales. Ello hace que el análisis presentado en este artículo cobre mayor relevancia, dado que constituye el primer estudio que explora la complementariedad entre estrategias de innovación tomando como muestra la industria manufacturera de un país tecnológicamente seguidor. La alta concentración de pequeñas y medianas empresas en sectores tradicionales de baja intensidad tecnológica o el bajo nivel de inversión empresarial en actividades de I+D (Bayona et al., 200I; Castro y Fernández, 2006), constituyen algunos rasgos distintivos del contexto español que pueden conducir a resultados diferentes de los encontrados en países desarrollados, los cuales tradicionalmente han sido el centro de la mayor parte de los estudios de esta naturaleza.

\section{Datos y metodología}

\section{Datos}

Los datos utilizados para el análisis empírico provienen del Panel de Innovación Tecnológica (PITEC) desarrollado conjuntamente por el Instituto Nacional de Estadística de España (INE), la Fundación Española para la Ciencia y la Tecnología (FECYT) y la Fundación Cotec ${ }^{7}$. La información recogida en PITEC está basada en las Encuestas de Innovación Tecnológica (EIT) e I+D que desde el año 2002 realiza de forma coordinada el INE, las cuales siguen la misma metodología empleada en las Encuestas comunitarias sobre innovación (CIS, por sus siglas en inglés) elaboradas por Eurostat. Para el año 2004, el PITEC cuenta aproximadamente con una muestra total de 4400 empresas manufactureras, distribuidas a lo largo del territorio español. No obstante, este artículo se focaliza en el colectivo de empresas activas en innovación. Estas empresas son las que contestan todas las preguntas del cuestionario, entre las que se encuentran aquellas relacionadas con la cooperación en actividades de innovación. La muestra definitiva, después de eliminar tanto las empresas no activas en innovación, como aquellas

\footnotetext{
${ }^{7}$ La base de datos generada se encuentra disponible públicamente en el portal de la FECYT (http://sise.fecyt.es/) y se presenta en un sistema de ficheros coordinados, a razón de un fichero por año cubierto por el panel, el cual se acompaña de toda la información necesaria para su interpretación y utilización.
}

ISSN: 07I 8-2724. (http://www.jotmi.org)

Journal of Technology Management \& Innovation () Universidad Alberto Hurtado, Facultad de Economía y Negocios 
observaciones indicadas en los ficheros como incidencias que pueden producir comparaciones anómalas (empresa de nueva creación, fusiones, venta o cierre de la empresa), es 3311 empresas, agrupadas en 28 sectores industriales diferentes de acuerdo a la Clasificación Nacional de Actividades Económicas (CNAE-1993).

\section{Metodología}

Tal como se ha mencionado anteriormente, este artículo parte de la distinción entre coexistencia y complementariedad como las dos dimensiones básicas para analizar las relaciones existentes entre las estrategias de innovación. Atendiendo a dicha distinción, el estudio empírico se estructura en dos grandes apartados.

En primer lugar, se lleva a cabo el análisis de la coexistencia, para lo cual se sigue el método propuesto por Arora y Gambardella (1990) basado en el cálculo de los coeficientes de correlación ${ }^{8}$. En la práctica, este método es implementado siguiendo dos pasos. En primer lugar, se estima una regresión para cada estrategia u práctica organizacional, utilizando como regresores las variables externas que puedan influir en la adopción de las mismas. En segundo lugar, se calculan los coeficientes de correlación entre las estrategias, utilizando para ello los residuos extraídos de las regresiones. Siguiendo este procedimiento se corrige el efecto que pueden ejercen variables exógenas observables sobre la correlación entre las estrategias, ofreciendo resultados más fiables que los que se obtienen al realizar un análisis de correlación normal.

El análisis de los determinantes de las estrategias de innovación (primer paso para el análisis de la coexistencia) es realizado través de la estimación del siguiente modelo econométrico:

\footnotetext{
${ }^{8}$ Cabe destacar que los autores desarrollaron este método como una técnica alternativa para evaluar la complementariedad, sugiriendo que si dos actividades son complementarias, la adopción de una incrementa la probabilidad de llevar a cabo la otra, por lo que existe una fuerte correlación positiva entre ellas, la cual se mantiene incluso después de controlar el efecto de características exógenas observables. Aunque posteriormente los mismos autores reconocieron las limitaciones de este método como prueba de complementariedad (Arora, 1996), lo cierto es que el mismo puede ser considerado un método fiable para determinar la coexistencia entre las estrategias.
}

Estrategia ${ }_{i}^{d}=\alpha_{0}+\alpha_{1}$ Lncifra $_{i}+\alpha_{2}$ Gruponal $_{i}+\alpha_{3}$ Grupo int er $r_{i}+$

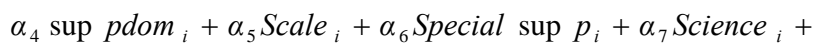
$\alpha_{8}$ Face $_{i}+\alpha_{9}$ Facorg $_{i}+\alpha_{10}$ Facmerc $+\alpha_{11}$ Spillinst $_{i}+$

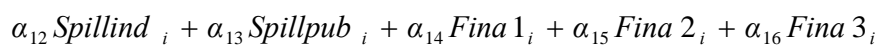

Donde $i=1, \ldots n$ (número de observaciones); $D=1, \ldots 6($ estrategias de innovación)

La variable dependiente (Estrategia) representa las diferentes estrategias de innovación consideradas en el análisis: I+D interna (Idint), I+D externa (Idext), compra de maquinarias y equipos (Maqui), adquisición de tecnología inmaterial (Tecno), cooperación con agentes industriales (Cind) y cooperación con agentes institucionales (Cinst). Todas son variables dicotómicas que toman el valor de I si la empresa ha llevado a cabo la estrategia en cuestión durante el periodo 2002-2004 y el valor de 0 en caso contrario.

Entre los regresores incluidos en el modelo se encuentran una serie de variables asociadas tanto con características empresariales, como industriales. En el caso de las características empresariales, se considera el tamaño de la empresa (Lncifra) y la pertenencia a un grupo empresarial (Gruponal y Grupointer). El efecto de la industria, por su parte, es valorado teniendo en cuenta la taxonomía de sectores industriales propuesta por Pavitt (1984), la cual distingue entre: empresas dominadas por los proveedores (Suppdom), las empresas de escala intensiva (Scale), los proveedores especializados (Specialsupp) y las empresas basadas en la ciencia (Science). Adicionalmente, se incluye un conjunto de variables que miden la importancia que la empresa otorga a una serie de factores como obstáculos para el desarrollo de actividades de innovación (Face, Facorg. Facmer), los spillovers derivados de diferentes fuentes externas (Spillinst, Spillind, Spillpub) y la obtención de apoyo financiero derivado de organismos públicos de carácter local, nacional y europeo (Final, Fina2, Fina3).

La segunda etapa del estudio empírico está orientada al análisis de la complementariedad. Para ello, se sigue el denominado enfoque de la función de producción, el cual constituye una aplicación más directa de la teoría de la supermodularidad propuesta por Milgrom y Roberts (1990). En términos generales este enfoque se basa en la estimación de una regresión, la cual contempla como variable dependiente una medida de productividad y como variables explicativas los efectos interactivos de las prácticas

ISSN: 07I 8-2724. (http://www.jotmi.org)

Journal of Technology Management \& Innovation (C) Universidad Alberto Hurtado, Facultad de Economía y Negocios 
analizadas, así como un conjunto de factores exógenos que pueden incidir sobre la función de desempeño. Con base a los resultados de dicha regresión se calcula un parámetro de complementariedad, el cual, en función de su signo y su significancia estadística, permite determinar si las prácticas analizadas son complementarias o sustitutivas.

En el caso de que se analice la complementariedad entre dos prácticas, la función de producción a estimar puede adoptar la siguiente forma:

$\left(A_{1}, A_{2}, X ;,\right)=\left(I-A_{1}\right)\left(I-A_{2}\right) 00+A_{1}\left(I-A_{2}\right) \quad 10+\left(I-A_{1}\right) A_{2}$ ${ }_{01}+A_{1} A_{2} 11+X+$

Donde $X$ representa el vector de variables exógenas que afectan la función de desempeño y los ij representan los coeficientes de las diferentes combinaciones de estrategias o prácticas organizacionales implementas por la empresa. En el caso de dos prácticas $\left(A_{1}, A_{2}\right)$, se obtienen las siguientes cuatro posibles combinaciones: a) adoptar las dos prácticas $(\mathrm{I}, \mathrm{I})$; b) adoptar solo $A_{1}(\mathrm{I}, 0)$; c) adoptar solo $A_{2}(0, \mathrm{I})$ y; d) no adoptar ninguna práctica $(0,0)$.

Atendiendo a la teoría de la supermodularidad, las prácticas $A_{1}$ y $A_{2}$ son complementarias si y solo si:

$$
(I, 0)+(0, I) \leq(I, I)+(0,0)
$$

Es decir, la suma de los cambios en la función de producción cuando las dos actividades son desarrolladas de forma separada es menor que el cambio resultante al desarrollar las dos actividades de forma conjunta. La expresión anterior es equivalente a:

$$
(I, I)-(0, I) \geq(I, 0)-(0,0)
$$

Esta última desigualdad da una idea más intuitiva del concepto de complementariedad anteriormente expuesto y supone que llevar a cabo una actividad $\left(A_{l}=1\right)$ cuando la otra está desempeñándose $\left(A_{2}=l\right)$ tiene un mayor efecto marginal sobre la función de desempeño () que llevar a cabo dicha actividad de forma aislada?

Para llevar a cabo el método anteriormente expuesto, se parte de la siguiente especificación econométrica general:

\footnotetext{
${ }^{9}$ El concepto de sustitución es similar al de complementariedad, con la diferencia que en lugar de obtener efectos marginales "mayores" al desarrollar las dos practicas de forma simultánea, lo que se tiene son efectos marginales "menores".
}

INNOV $_{i}^{d}=\alpha_{0}+\alpha_{1}$ Lncifra $_{i}+\alpha_{2}$ Idext $_{i}+\alpha_{3}$ Maqui $_{i}+\alpha_{4}$ Tecno $_{i}+$ $\alpha_{5}$ Cinst $_{i}+\alpha_{6}$ Cind $_{i}+\alpha_{7}$ Id int $_{i}+\alpha_{8}$ Form $_{i}+\alpha_{9}{\text { Id } \text { int }_{i}}^{*}$ Cinst $_{i}+$

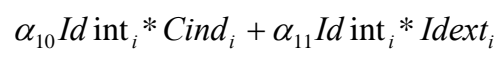

Donde $i=I, \ldots, N$ (número de observaciones)

La variable INNOV constituye la medida de desempeño empleada para evaluar la complementariedad entre las estrategias de innovación y representa la introducción de productos nuevos en el mercado. En concreto, ésta variable toma el valor de I, si la empresa ha introducido un producto nuevo en el mercado durante el periodo 20022004, y el valor 0 en caso contrario. Siguiendo el enfoque de la función de producción en el modelo se incluyen un conjunto de términos interactivos que representan las estrategias sobre las cuales se desea analizar la existencia de complementariedad. Específicamente, estos términos son calculados como el producto obtenido al multiplicar la variable Idint por las variables Idext, Cinst, Cind ${ }^{10}$. Adicionalmente, se incluyen como represores el tamaño de la empresa (Lncifra) y tres variables asociadas con el desarrollo de otras estrategias de innovación: Maqui, Tecno y Form".

\section{Resultados y discusión}

La tabla I muestra los resultados del análisis de correlación, el cual se ha realizado teniendo en cuenta los residuos obtenidos a partir de la estimación los modelos referidos a los determinantes de las diferentes estrategias de innovación ${ }^{12}$.

\footnotetext{
${ }^{10}$ Es importante señalar que, tal como se encuentra definido el modelo econométrico, la complementariedad entre las estrategias de innovación es evaluada partiendo de la hipótesis general derivada de la teoría de la capacidad de absorción, es decir, analizando si las actividades internas de I+D incrementan la capacidad de la empresa para explotar el conocimiento externo en el desarrollo de innovaciones. En este sentido, el análisis de la complementariedad entre estrategias asociadas únicamente a la adquisición de conocimiento externo (p.e. entre comprar y cooperar) se encuentra fuera del alcance de esta investigación. " La variable Form toma el valor de I si la empresa ha llevado a cabo actividades de formación orientadas hacia la innovación durante el periodo 2002-2004 y el valor de 0 en caso contrario. 12 Teniendo en cuenta el carácter dicotómico de las variables empleadas para evaluar el uso de las estrategias de innovación, las 6 estimaciones del modelo I (una para cada estrategia) se han realizado utilizando como técnica de análisis la regresión logística binaria. Dado que el análisis de los determinantes de las estrategias de innovación no es el objetivo de este trabajo y,
}

ISSN: 07I 8-2724. (http://www.jotmi.org)

Journal of Technology Management \& Innovation (C) Universidad Alberto Hurtado, Facultad de Economía y Negocios 


\begin{tabular}{|c|c|c|c|c|c|}
\hline & Idint & Idext & Maqui & Tecno & Cinst \\
\hline Idint & I & & & & \\
\hline Idext & $-0.082 * *$ & I & & & \\
\hline Maqui & -0.002 & $0.142 * *$ & I & & \\
\hline Tecno & $0.157 * *$ & $0.158 * *$ & $0.246 * *$ & I & \\
\hline Cinst & $0.078 * *$ & $0.208 * *$ & $0.050 * *$ & $0.126 * *$ & I \\
\hline Cind & $0.066 * *$ & $0.190 * *$ & $0.123 * *$ & $0.204 * *$ & $0.425^{* *}$ \\
\hline \multicolumn{6}{|c|}{ **La correlación es significativa al nivel 0.01 (bilateral) } \\
\hline & \multicolumn{5}{|c|}{$\mathrm{N}=33$ I I observaciones } \\
\hline
\end{tabular}

Tabla I. Correlación entre las estrategias de innovación (análisis de la coexistencia)

Tal como se observa, todas las estrategias asociadas con la adquisición externa de conocimiento están positiva y significativamente correlacionadas entre sí. Este resultado sugiere que una vez que la empresa ha decidido abrirse a la búsqueda externa de conocimiento, a través de la implementación de una estrategia en concreto, es mucho más probable que adopte cualquier otra estrategia de este tipo.

Las relaciones entre las estrategias asociadas con la generación interna y la adquisición externa de conocimiento muestran patrones interesantes. Por una parte, la variable Idint está negativa y significativamente correlacionada con la variable Idext, pero, por otra parte, se encuentra positiva y significativamente correlacionada con las variables Tecno, Cind y Cinst. El primer resultado sugiere que, para las empresas españolas, el desarrollo y la compra de I+D constituyen básicamente opciones alternativas. Es decir, las empresas eligen entre una u otra, siendo menos probable la adopción conjunta de las mismas.

Por el contrario, el segundo resultado indica que las empresas que realizan actividades internas de I+D están más propensas a adquirir tecnología inmaterial $\circ$ a cooperar con otros agentes en actividades de innovación. Este resultado puede interpretarse como una evidencia a

considerando las limitaciones de espacio, los resultados de dichas regresiones no se presentan en este artículo. favor de la teoría de la capacidad de absorción o, por lo menos, a una parte de ella ${ }^{13}$.

En lo que respecta al análisis de la complementariedad, la estimación del segundo modelo econométrico se ha realizado no sobre el total de empresas manufactureras, sino considerando dos submuestras conformadas por las empresas dominadas por los proveedores y por las empresas basadas en la ciencia. La decisión de focalizar la atención en estas dos categorías obedece a que las mismas son las que presentan los patrones de innovación más diferenciados y son en las que se distinguen con mayor claridad tanto la utilización de las diferentes estrategias de innovación, como la importancia de las fuentes de conocimiento industrial e institucional (Vega-Jurado et al., 2008). En este sentido, el análisis de estas categorías permitirá examinar si la complementariedad entre las estrategias de innovación es más fuerte en aquellos sectores que tienen una mayor dependencia hacia el knowhow "básico" (Cassiman y Veugelers, 2006), o en general, si la adquisición externa de conocimiento es una estrategia más importante para estimular el desempeño innovador en aquellos sectores donde la complejidad tecnológica es mayor.

Las tablas 2 y 3 muestran los resultados para las empresas dominadas por los proveedores y para las empresas basadas en la ciencia, respectivamente. Para evitar problemas de multicolinealidad, los tres términos interactivos considerados en el análisis son introducidos uno a uno, lo que da lugar a las tres estimaciones reportadas en cada una de las tablas. Dado el carácter dicotómico de la variable dependiente, la técnica de estimación empelada ha sido la regresión logística binaria ${ }^{14}$. En todos los casos, los valores Chi-cuadrado para los grados de libertad correspondientes sugieren el rechazo de la hipótesis nula de que todos los parámetros,

13 Para Cohen y Levinthal (1990), la capacidad de absorción no sólo facilita la identificación y la adquisición del conocimiento externo relevante, sino que además promueve su explotación. El análisis de correlación aquí desarrollado puede ofrecer evidencia empírica sobre los dos primeros aspectos, pero no sobre el último. El efecto asociado a la explotación del conocimiento, puede ser explorado a través del análisis de complementariedad.

${ }^{14}$ En la estimación del modelo se ha incluido además la variable Prob, la cual representa la probabilidad pronosticada de que una empresa sea activa en innovación. Esta variable se ha incluido con el objetivo de controlar el posible sesgo de selección derivado de realizar el análisis únicamente sobre el colectivo de empresas activas en innovación (vega-Jurado et al., 2009).

ISSN: 07I 8-2724. (http://www.jotmi.org)

Journal of Technology Management \& Innovation () Universidad Alberto Hurtado, Facultad de Economía y Negocios 
exceptuando la intersección, son iguales a cero con un nivel de significancia del $\mathrm{l} \%$.

Antes de revisar los resultados asociados con la complementariedad, vale la pena destacar algunos patrones interesantes con relación al efecto de las estrategias de innovación. En primer lugar, se observa que la contratación de actividades externas de I+D (Idext) no constituye una estrategia importante para el desarrollo de nuevos productos, ni siquiera para las empresas basadas en la ciencia. De hecho, los coeficientes estimados para esta variable fueron negativos en todos los casos. Asimismo, los resultados ponen de manifiesto que, si bien la cooperación con agentes externos influye sobre el desempeño innovador de la empresa, el socio adecuado puede ser muy diferente atendiendo al sector industrial en el que opera la empresa. En línea con lo planteado en la literatura, para las empresas dominadas por los proveedores la cooperación con agentes industriales es una estrategia importante, mientras que para las empresas basadas en la ciencia, únicamente es significativa la cooperación con agentes institucionales.

La estimación de cada uno términos interactivos se ha realizado teniendo en cuenta las diferentes combinaciones posibles y empleando como referencia el caso asociado con el desarrollo conjunto de las dos estrategias, es decir, cuando $A_{1}=1$ y $A_{2}=1$. En lo que se refiere a la cooperación con agentes externos (industrial e institucional), los signos negativos de todos los coeficientes estimados sugieren que la articulación de dicha estrategia con el desarrollo interno de actividades de I+D supone, por lo general, mejores resultados. Por el contrario, en el caso de la I+D externa, se observa el patrón inverso. Estos resultados, están en línea con los señalados anteriormente y son un reflejo de los efectos principales de dichas variables. Es decir, corroboran el efecto positivo de la cooperación, institucional o industrial, según sea la categoría sectorial analizada, y el efecto negativo de la contratación de actividades de I+D externa. Sin embargo, estos resultados no aportan evidencia sobre la complementariedad o sustitución entre dichas estrategias y el desarrollo de actividades internas de I+D. Para analizar este último aspecto, se han calculado los efectos marginales de cada uno de los términos interactivos, manteniendo el resto de las co-variables en sus valores promedio. Los resultados son presentados en las tablas 4 y 5 , junto con el test de complementariedad.

\begin{tabular}{|c|c|c|c|}
\hline Variables independientes & Estimación I & Estimación 2 & Estimación 3 \\
\hline (Constante) & $-0,83 \quad(1,02)$ & $-0,61 \quad(1,05)$ & $-I, 40 \quad(I, 0 I)$ \\
\hline Lncifra & $0,05 \quad(0,06)$ & $0,05 \quad(0,06)$ & $0,05(0,06)$ \\
\hline Prob & $\mathrm{I}, 3 \mathrm{I} * * \quad(0,6 \mathrm{I})$ & $1,29 * * \quad(0,61)$ & $1,30^{* *}(0,61)$ \\
\hline Tecno & $0,5 I^{*}(0,29)$ & $0,50 * \quad(0,29)$ & $0,50 *(0,29)$ \\
\hline Cind & $0,57^{* *} \quad(0,27)$ & & 0,56 ** $(0,27)$ \\
\hline Cinst & & $0,43 \quad(0,27)$ & $0,44 \quad(0,27)$ \\
\hline Idext & $-0,16 \quad(0,18)$ & $-0,16 \quad(0,18)$ & \\
\hline Form & $0,10 \quad(0,20)$ & $0,10 \quad(0,20)$ & $0, I I \quad(0,20)$ \\
\hline Maqui & $0,15 \quad(0,19)$ & $0,14 \quad(0,19)$ & $0,15 \quad(0,19)$ \\
\hline$[$ Idint $=0] *[$ Cinst $=0]$ & $-1,34 * * * \quad(0,33)$ & & \\
\hline$[$ Idint $=0] *[$ Cinst $=I]$ & $-0,68 \quad(0,55)$ & & \\
\hline$[$ Idint $=I] *[$ Cinst $=0]$ & $-0,36 \quad(0,31)$ & & \\
\hline \multicolumn{4}{|l|}{$[$ Idint $=I] *[$ Cinst $=I]$} \\
\hline$[$ Idint $=0] *[$ Cind $=0]$ & & $-1,51 * * * \quad(0,35)$ & \\
\hline$[$ Idint $=0] *[$ Cind $=I]$ & & $-0,97^{* *}(0,49)$ & \\
\hline$[\operatorname{ldin} t=I] *[$ Cind $=0]$ & & $-0,57 *(0,32)$ & \\
\hline \multicolumn{4}{|l|}{$[\operatorname{ldint}=1] *[$ Cind $=I]$} \\
\hline$[$ Idint $=0] *[$ Idext $=0]$ & & & $-0,83 * * * \quad(0,31)$ \\
\hline
\end{tabular}

ISSN: 07I 8-2724. (http://www.jotmi.org)

Journal of Technology Management \& Innovation () Universidad Alberto Hurtado, Facultad de Economía y Negocios 


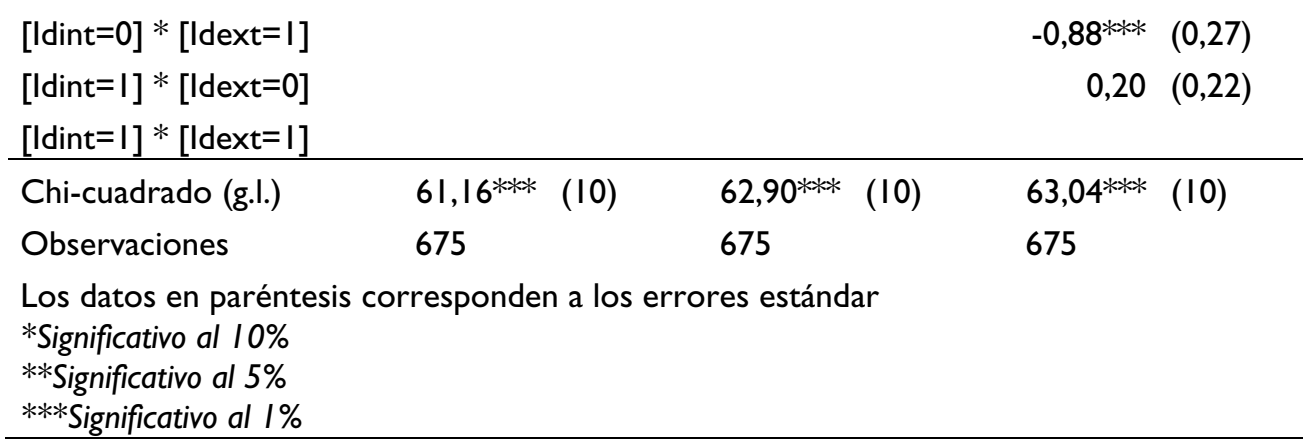

Tabla 2. Efecto de las estrategias de innovación sobre la innovación de producto. Empresas dominadas por los proveedores

\begin{tabular}{|c|c|c|c|}
\hline $\begin{array}{l}\text { Variables } \\
\text { independientes }\end{array}$ & Estimación I & Estimación 2 & Estimación 3 \\
\hline (Constante) & $-0,34 \quad(1,32)$ & $-0,36 \quad(1,34)$ & $-0,97 \quad(1,32)$ \\
\hline Lncifra & $-0,05 \quad(0,06)$ & $-0,05 \quad(0,06)$ & $-0,05 \quad(0,06)$ \\
\hline Prob & $2,73^{* *}(1,14)$ & $2,75^{* *}(1,14)$ & $2,77 * * \quad(I, \mid 4)$ \\
\hline Tecno & $0,26 \quad(0,33)$ & $0,25 \quad(0,33)$ & $0,28 \quad(0,33)$ \\
\hline Cind & $0,42 \quad(0,26)$ & & $0,43 \quad(0,26)$ \\
\hline Cinst & & $0,46^{*} \quad(0,26)$ & $0,46 *(0,26)$ \\
\hline Idext & $-0,13 \quad(0,21)$ & $-0,13 \quad(0,21)$ & \\
\hline Form & $0,02 \quad(0,2 \mathrm{I})$ & $0,0 \mathrm{I} \quad(0,2 \mathrm{I})$ & $0,02 \quad(0,2 I)$ \\
\hline Maqui & 0,50 ** $\quad(0,22)$ & $0,51 * * \quad(0,22)$ & $0,53^{* *} \quad(0,22)$ \\
\hline$[$ Idint $=0] *[$ Cinst $=0]$ & $-1,49 * * * \quad(0,42)$ & & \\
\hline$[$ Idint $=0] *[$ Cinst $=I]$ & $-0,84 \quad(0,86)$ & & \\
\hline$[$ Idint $=I] *[$ Cinst $=0]$ & $-0,43 \quad(0,27)$ & & \\
\hline \multicolumn{4}{|l|}{$[\operatorname{ldin} t=I] *[$ Cinst $=I]$} \\
\hline$[$ Idint $=0] *[$ Cind $=0]$ & & $-1,53 * * * \quad(0,45)$ & \\
\hline$[$ Idint $=0] *[$ Cind $=I]$ & & $-0,57 \quad(0,74)$ & \\
\hline$[$ Idint $=I] *[$ Cind $=0]$ & & $-0,36 \quad(0,27)$ & \\
\hline \multicolumn{4}{|l|}{$[\operatorname{ldin} t=I] *[$ Cind $=I]$} \\
\hline$[$ Idint $=0] *[$ ldext $=0]$ & & & $-1,16 * * \quad(0,52)$ \\
\hline$[$ Idint $=0] *[$ Idext $=I]$ & & & $-0,77^{*} \quad(0,45)$ \\
\hline$[$ Idint $=I] *[$ Idext $=0]$ & & & $0,20 \quad(0,23)$ \\
\hline \multicolumn{4}{|l|}{$[\operatorname{ldin} \mathrm{t}=\mathrm{I}] *[\operatorname{ldex} \mathrm{t}=\mathrm{I}]$} \\
\hline Chi-cuadrado (g.l.) & 40,5 I*** $(10)$ & $40,99 * * * \quad(10)$ & $41,20 * * * \quad(10)$ \\
\hline Observaciones & 654 & 654 & 654 \\
\hline $\begin{array}{l}\text { Los datos en paréntes } \\
\text { *Significativo al } 10 \% \\
\text { **Significativo al } 5 \% \\
\text { ***Significativo al } 1 \%\end{array}$ & corresponden a lo & errores estándar & \\
\hline
\end{tabular}

Tabla 3. Efecto de las estrategias de innovación sobre la innovación de producto. Empresas basadas en la ciencia 


\begin{tabular}{|c|c|c|c|c|c|}
\hline & \multirow[b]{3}{*}{$\left(A_{1}, A_{2}\right)$} & \multirow[b]{3}{*}{ Media Marginal } & \multicolumn{3}{|c|}{ Test de complementariedad } \\
\hline & & & \multicolumn{2}{|c|}{$(1,1)-(1,0)>(0,1)-(0,0)$} & \multirow{2}{*}{$\begin{array}{l}\text { Significancia } \\
\mathrm{t} \text { (g.l.) }\end{array}$} \\
\hline & & & $(1,1)-(1,0)$ & $(0,1)-(0,0)$ & \\
\hline \multirow{4}{*}{$\begin{array}{l}\text { Idint }\left(A_{1}\right)^{*} \\
\text { Cinst }\left(A_{2}\right)\end{array}$} & $(1,1)$ & 0,78 & \multirow{2}{*}{0,07} & & \multirow{4}{*}{$2,34 * *(673)$} \\
\hline & $(1,0)$ & 0,71 & & & \\
\hline & $(0,1)$ & 0,64 & \multirow{2}{*}{\multicolumn{2}{|c|}{0,16}} & \\
\hline & $(0,0)$ & 0,48 & & & \\
\hline \multirow{4}{*}{$\begin{array}{l}\text { Idint }\left(A_{1}\right) * \\
\text { Cind }\left(A_{2}\right)\end{array}$} & $(1,1)$ & 0,81 & \multirow{4}{*}{0,11} & & \multirow{4}{*}{$0,52(673)$} \\
\hline & $(1,0)$ & 0,7 & & & \\
\hline & $(0,1)$ & 0,61 & & \multirow{2}{*}{0,13} & \\
\hline & $(0,0)$ & 0,48 & & & \\
\hline \multirow{4}{*}{$\begin{array}{l}\operatorname{Idint}\left(A_{1}\right)^{*} \\
\operatorname{Idext}\left(A_{2}\right)\end{array}$} & $(1,1)$ & 0,71 & \multirow{4}{*}{$-0,04$} & & \multirow{4}{*}{$0,78(673)$} \\
\hline & $(1,0)$ & 0,75 & & \multirow{3}{*}{$-0,01$} & \\
\hline & $(0,1)$ & 0,5 & & & \\
\hline & $(0,0)$ & 0,51 & & & \\
\hline
\end{tabular}

Tabla 4. Test de complementariedad. Empresas dominadas por los proveedores

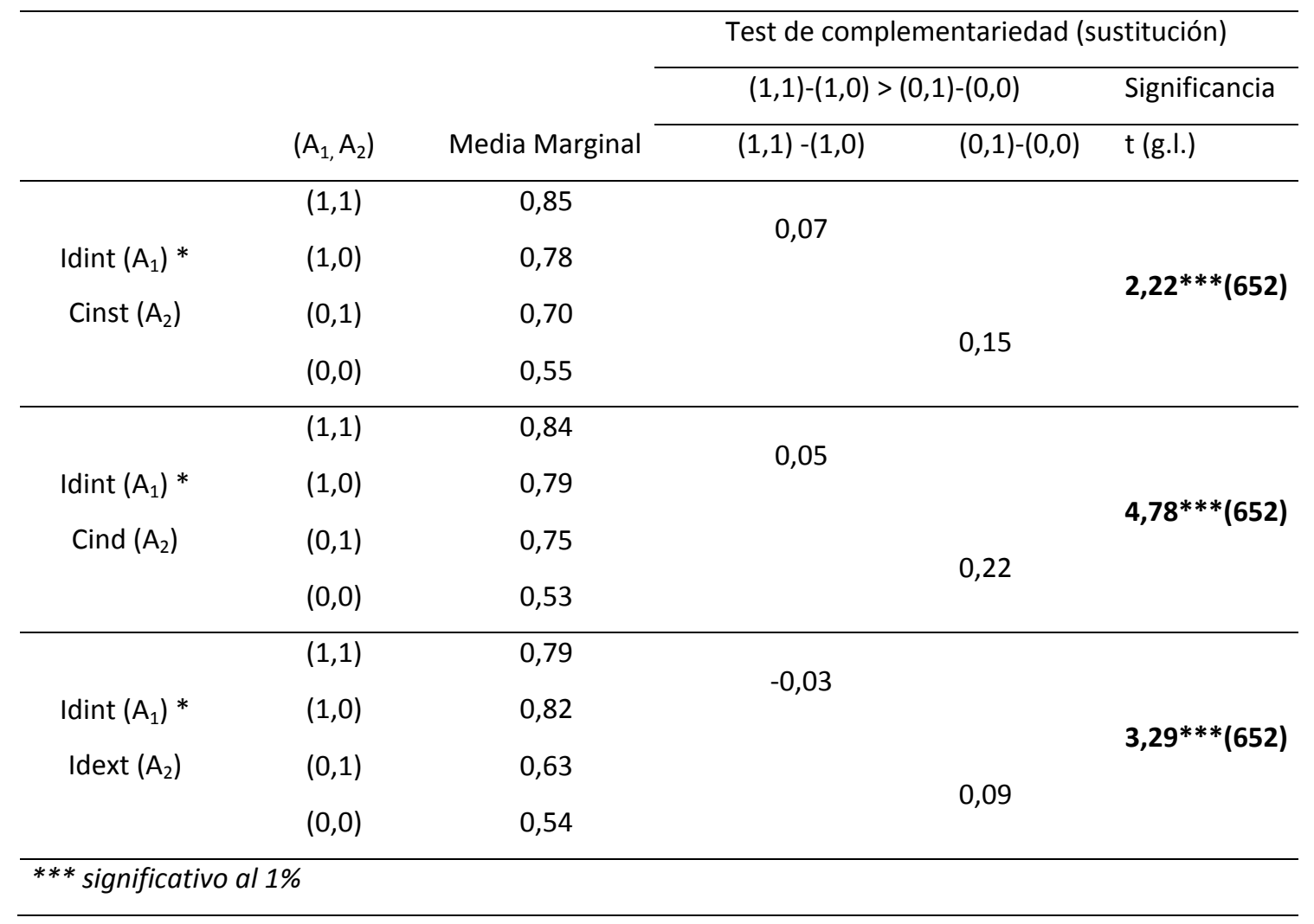

Tabla 5. Test de complementariedad. Empresas basadas en la ciencia 
Tal como se observa, en ninguna de las dos categorías sectoriales analizadas y para ninguna de las combinaciones de estrategias consideradas en este análisis, se encuentra evidencia de complementariedad. De hecho, en todos los casos el rendimiento marginal que se obtiene al llevar a cabo una actividad cuando la otra está realizándose, es menor que el efecto marginal que se obtiene cuando dicha actividad se lleva a cabo de forma aislada ${ }^{15}$. En otras palabras, lo que ponen de manifiesto los resultados obtenidos es una posible relación de sustitución entre las estrategias analizadas, la cual es, además, en todos los casos significativa para el colectivo de empresas basadas en la ciencia.

Las figuras I y 2 muestran gráficamente los resultados obtenidos para las empresas dominadas por los proveedores y para las empresas basadas en la ciencia, respectivamente. En general, se puede observar que conforme las empresas llevan a cabo actividades internas de I+D (la variable Idint pasa de 0 a I), la pendiente de las rectas que representan el efecto marginal de las diferentes estrategias de adquisición de conocimiento externo sobre la innovación de producto disminuye ${ }^{16}$. Este resultado sugiere que cuando las empresas llevan a cabo actividades internas de $I+D$, los efectos marginales que ejerce tanto la cooperación con agentes externos (industriales e institucionales), como la contratación de actividades externas de I+D sobre la innovación de producto tienden a ser menor.

En resumen, los resultados no ofrecen evidencia a favor de que un nivel alto de capacidades tecnológicas internas, derivadas del desarrollo de actividades de I+D, incrementen la explotación del conocimiento externo para el desarrollo de nuevos productos. Estos resultados son contrarios a los encontrados por Cassiman y Veugelers (2006) para el caso de las empresas belgas, pero están en la línea con los hallazgos de Laursen y Salter (2006) sobre la existencia de efectos sustitutivos entre las actividades internas de I+D y el uso de fuentes externas de conocimiento. La explicación que estos

${ }^{15}$ En todos los casos se encontró que: $(1,1)-(1,0)$ es menor que: $(0,1)-(0,0)$

${ }^{16}$ En el caso de las empresas dominadas por los proveedores, cuando la variable Idint cambia de 0 a 2, la pendiente de la recta disminuye desde 0,06 a 0,04 . Mientras que para las empresas basadas en la ciencia la pendiente de la recta disminuye desde 0,12 a 0,04 . últimos autores sugirieron para este resultado fue la del síndrome del "no inventado aquí", la cual puede ser igualmente válida para el caso analizado. La realización continua de actividades de I+D aumenta la capacidad tecnológica de la empresa y puede llevar a los responsables del departamento de I+D a pensar que dominan los conocimientos requeridos en su campo. Cuando ello ocurre la empresa se encuentra más renuente a aceptar ideas externas, especialmente aquellas que están más cercanas al campo de sus competencias nucleares. Lo anterior puede llevar a la empresa a cooperar menos con agentes externos o, alternativamente y tal como parece ocurrir en el caso analizado, a que la empresa centre la cooperación en actividades no orientadas directamente a fortalecer sus competencias innovadoras. Este fenómeno se agudiza en contextos como el español, donde por las características propias del tejido productivo, los agentes científicos, y en especial las universidades, no han sido consideradas por las empresas como una fuente de conocimiento importante para las actividades innovadoras. De hecho, un resultado que se deriva de la Encuesta de Innovación es que sólo el $8.2 \%$ de las empresas manufactureras le atribuyen a las universidades una importancia alta como fuente de conocimiento para el desarrollo de los procesos de innovación ${ }^{17}$. Este último argumento estaría en línea con el hallazgo de Cassiman y Veugelers (2006) con relación a que la confianza en knowhow de carácter básico afecta la fortaleza de la complementariedad entre las estrategias de innovación.

\footnotetext{
17 Por categoría sectorial estos porcentajes son: 13.5\% para las empresas basadas en la ciencia y $2.4 \%$ para las empresas dominadas por los proveedores.
}

ISSN: 07I 8-2724. (http://www.jotmi.org)

Journal of Technology Management \& Innovation () Universidad Alberto Hurtado, Facultad de Economía y Negocios 


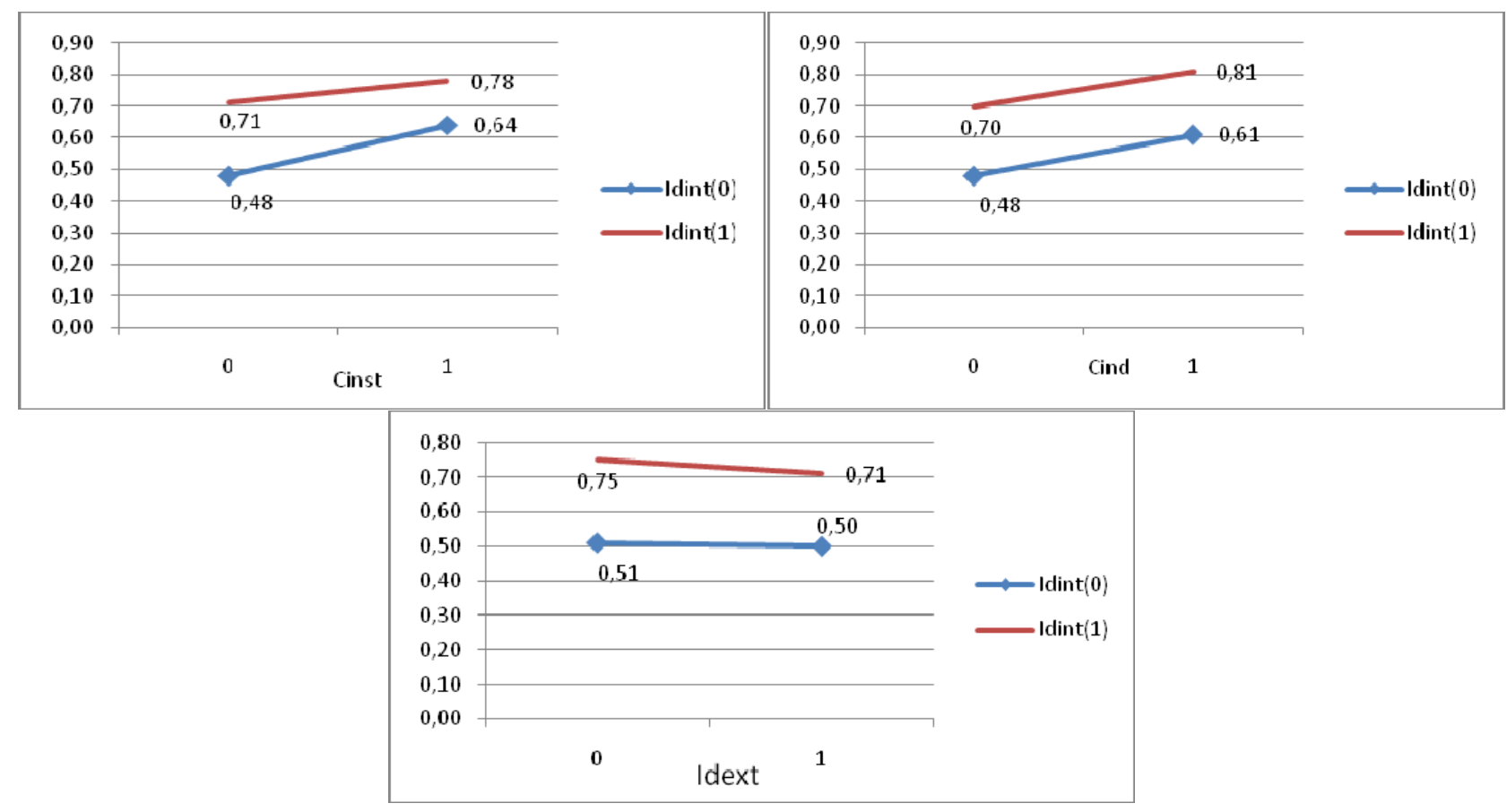

Figura I. Empresas dominadas por los proveedores

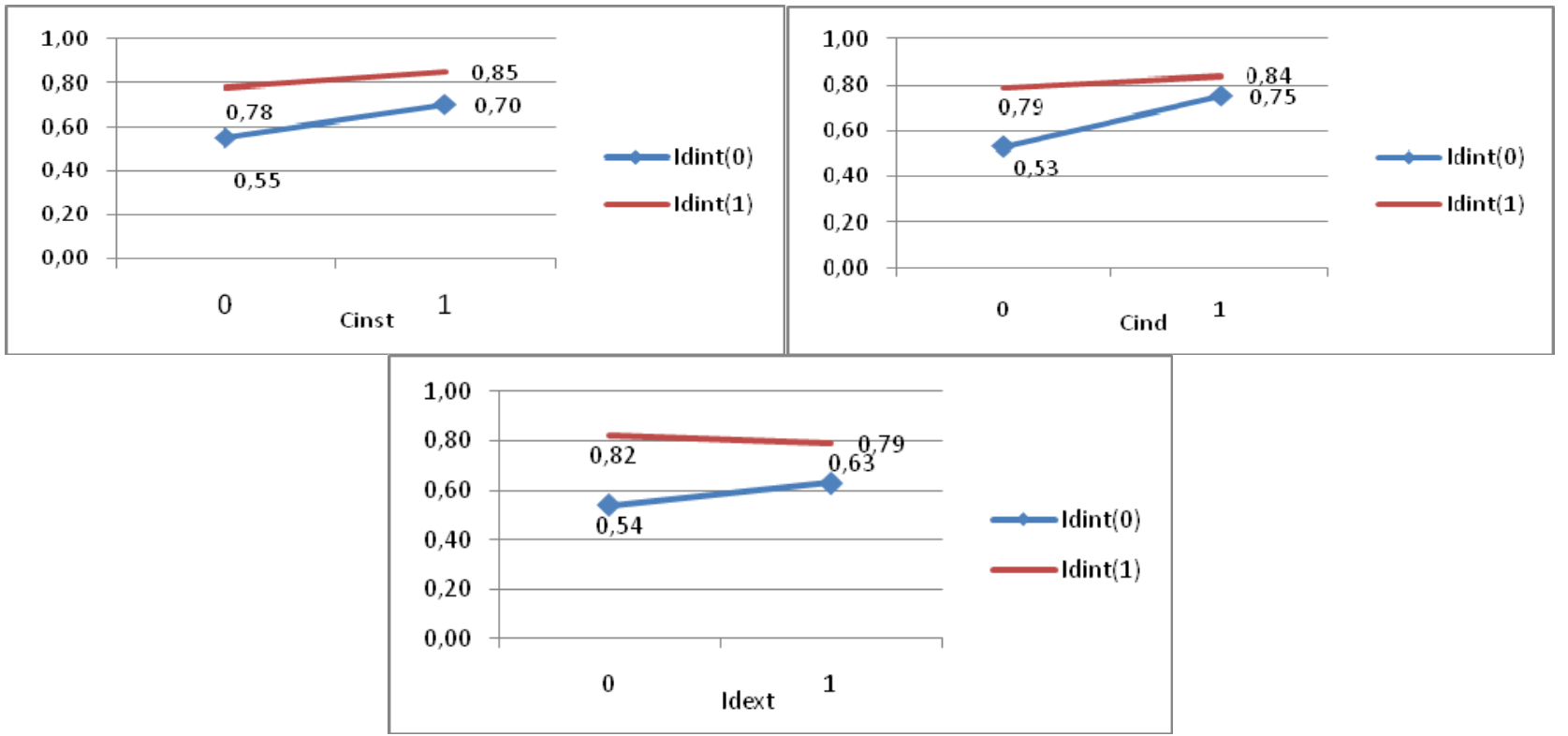

Figura 2. Empresas basadas en la ciencia

Considerando conjuntamente los resultados señalados anteriormente con aquellos derivados del análisis de correlación se observa un patrón interesante. Las empresas que llevan a cabo actividades internas de $\mathrm{I}+\mathrm{D}$, en comparación con aquellas que no las hacen, tienden a cooperar más con agentes institucionales, pero ello no implica que dicha cooperación sea más relevante para el desarrollo de nuevos productos. En otras palabras, aunque el desarrollo y la adquisición externa de conocimiento sean actividades que coexistan dentro de la estrategia global de

ISSN: 07I 8-2724. (http://www.jotmi.org)

Journal of Technology Management \& Innovation (@) Universidad Alberto Hurtado, Facultad de Economía y Negocios 
la empresa, ello no implica que existan efectos sinérgicos entre las mismas.

\section{Conclusiones}

En este artículo se han analizado las relaciones existentes entre la generación y la adquisición externa de conocimiento como estrategias de innovación empresarial. En términos generales, los resultados indican que para la industria manufacturera española las actividades internas de I+D y las estrategias asociadas con la adquisición externa de conocimiento son estrategias coexistentes, pero no complementarias. Esta premisa, aunque simple, tiene importantes implicaciones. En primer lugar, pone de manifiesto la necesidad de distinguir entre "coexistencia" y "complementariedad" como dos aspectos diferentes para el análisis de las relaciones entre las estrategias de innovación. Si bien estos aspectos pueden estar relacionados, es un error suponer que el primero implica el segundo, tal como se ha hecho en la mayoría de los estudios empíricos realizados hasta la fecha. En segundo lugar, este resultado destaca la importancia de considerar el carácter multidimensional de la capacidad de absorción, al momento de evaluar el papel que ejerce la I+D interna sobre el uso de las fuentes externas de conocimiento. En este sentido, una cosa es que las actividades internas de I+D faciliten la identificación y adquisición de conocimiento externo, aspecto ampliamente confirmado en este estudio, y otra que promuevan efectivamente la explotación de dicho conocimiento en el desarrollo de innovaciones. Tener en cuenta estos dos posibles efectos y evaluarlos empíricamente constituye un aspecto clave para avanzar en este campo de investigación.

Estos resultados también tienen una implicación política importante en el contexto español. Si el objetivo de las políticas de innovación es incentivar el uso de las fuentes externas de conocimiento, se puede decir que se está consiguiendo, especialmente a través del fomento de la cooperación. Ahora bien, ello no quiere decir que dicha estrategia sea eficiente para incentivar el desempeño innovador de las empresas. En este sentido, lo importante no es acceder a una gran diversidad de fuentes externas de conocimiento, sino acceder a aquéllas que realmente ofrezcan la posibilidad de desarrollar las capacidades innovadoras de la empresa a partir de la integración de conocimiento complementario. Para que ello suceda será necesario, por una parte, establecer instrumentos orientados específicamente a fortalecer las capacidades tecnológicas internas de la empresa $y$, por otra, definir instrumentos de fomento más selectivos en lo concerniente al uso de las fuentes externas de conocimiento.

\section{Referencias}

ARORA, A. (1996). Testing for complementarities in reduced-form regressions. Economics Letter 50, 5I-55.

ARORA, A., Gambardella, A. (1990). Complementarity and external linkages: the strategies of the large firms in biotechnology. Journal of Industrial Economics 38, 36I-379.

ARORA, A., Gambardella, A. (1994). Evaluating technological information and utilizing it: Scientific knowledge, technological capability and external linkages in biotechnology. Journal of Economic Behavior and Organization 24, $91-114$.

ATHEY, S., Stern, S. (1998). An empirical framework for testing theories about complementarity in organizational design. NBER working paper 6600.

BARNEY, J.B. (1991). Firm resources and sustained competitive advantage. Journal of Management I7, 99-120.

BAYONA, C., Garcia-Marco, T., Huerta, E. (200I). Firms' motivations for cooperative R\&D: an empirical analysis of Spanish firms. Research Policy 30, 1289-1307.

BECKER, W., Dietz, J. (2004). R\&D cooperation and innovation activities of firms-evidence for the German industry. Research Policy 33, 209-223.

CASSIMAN, B., Veugelers, R. (2006). In search of complementarity in innovation strategy: internal R\&D and external knowledge acquisition. Management Science 52, 68-82.

CASTRO, E., Fernández-de-Lucio, I. (2006). La I+D empresarial y sus relaciones con la investigación pública española. En: Sebastián, J., Muñoz, E. (eds.), Radiografia de la investigación pública en España. Biblioteca Nueva, Madrid.

COHEN, W., Levinthal, D. (1990). Absorptive Capacity: A new perspective on learning and innovation. Administrative Science Quarterly 35, I28-I52.

FORAY, D., Mowery, D (1990). L'integration de la R\&D industrielle: nouvelles perspectives d'analyse. Reveu Economique 3.

ISSN: 07 I 8-2724. (http://www.jotmi.org)

Journal of Technology Management \& Innovation (C) Universidad Alberto Hurtado, Facultad de Economía y Negocios 
FREEMAN, C. (1991). Networks of innovators: a synthesis of research issues. Research Policy 20, 499-5I4

HOETKER, G. (2007). The use of logit and probit models in strategic management research: critical issues. Strategic Management Journal 28, 33I-453.

KAISER, U. (2002). An empirical test of models explaining research expenditures and research cooperation: evidence for the German service sector. Research Policy 20, 747-774.

LAURSEN, K., Salter, A. (2006). Open for Innovation: The role of openness in explaining innovative performance among U.K. manufacturing firms. Strategic Management Journal 27, I3I-I50.

LOWE, J., Taylor, P. (1998). R\&D and technology purchase through licence agreements: complementary strategies and complementary assets. R\&D Management 28, (4), 263-278.

MILGROM, P., Roberts, J. (1990). The economics of modern manufacturing: Technology, strategy, and organization. American Economic Review 80, 5 I I-528.

MOWERY, D.C. (1983). The relationship between intrafirm and contractual forms of industrial research in
American manufacturing, 1900-1940. Exploration in Economics History 20, 35I-374.

MOWERY, D.C., Rosenberg, N. (1989), Technology and the Pursuit of Economic Growth. Cambridge University Press, Cambridge.

PAVITT, K. (1984). Sectoral patterns of technical change. Research Policy 13, 343-373

TEECE D., Pisano G., Shuen A. (1997). Dynamic capabilities and strategic management. Strategic Management Journal 18 , (7), 509-533.

VEGA-JURADO, J., Gutierrez-Gracia, A., Fernández-deLucio, I., Manjarrés-Henríquez, L. (2008a). The effect of external and internal factors on firms' product innovation. Research Policy 37, 616-632.

VEGA-JURADO, J., Gutiérrez Gracia, A., Fernández de Lucio, I. (2008b). ¿Cómo innovan las empresas españolas? Una evidencia empírica. Journal of Technology Management \& Innovation 3, 100-III

VEUGELERS, R. (1997). Internal R\&D expenditures and external technology sourcing. Research Policy 26, 303-3I

ISSN: 07| 8-2724. (http://www.jotmi.org)

Journal of Technology Management \& Innovation ( Universidad Alberto Hurtado, Facultad de Economía y Negocios 